\title{
Formation of Silicene Nanosheets on Graphite
}

\author{
Maurizio De Crescenzi, ${ }^{*}{ }^{\dagger}$ (i) Isabelle Berbezier, ${ }^{*}{ }^{\ddagger}$ Manuela Scarselli, $^{\dagger}$ Paola Castrucci, ${ }^{\dagger}$ Marco Abbarchi, $^{\dagger}$ \\ Antoine Ronda, ${ }^{\ddagger}$ Fatme Jardali, ${ }^{\S}$ Jejune Park, $^{\S}$ and Holger Vach*,§ \\ †Dipartimento di Fisica, Università di Roma “Tor Vergata”, 00133 Roma, Italy \\ "CNRS, Aix-Marseille Université, IM2NP, UMR 7334, Campus de St. Jérome, 13397 Marseille, France \\ ${ }^{\S}$ CNRS-LPICM, Ecole Polytechnique, Université Paris-Saclay, 91128 Palaiseau, France
}

Supporting Information

\begin{abstract}
The extraordinary properties of graphene have spurred huge interest in the experimental realization of a two-dimensional honeycomb lattice of silicon, namely, silicene. However, its synthesis on supporting substrates remains a challenging issue. Recently, strong doubts against the possibility of synthesizing silicene on metallic substrates have been brought forward because of the non-negligible interaction between silicon and metal atoms. To solve the growth problems, we directly deposited silicon on a chemically inert graphite substrate at room temperature.
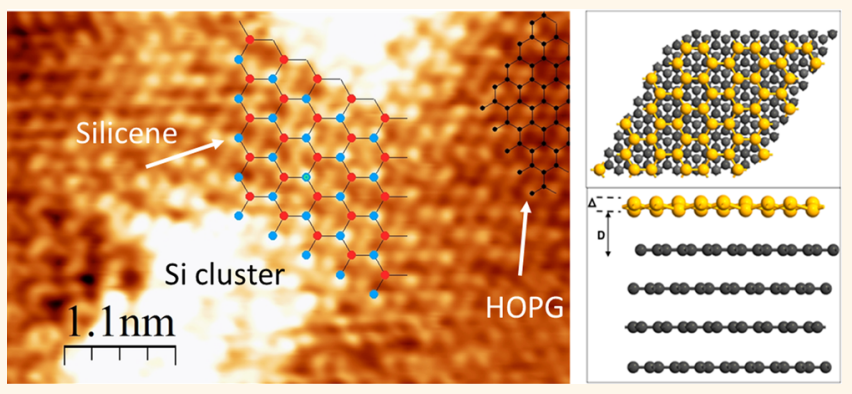
Based on atomic force microscopy, scanning tunneling microscopy, and $a b$ initio molecular dynamics simulations, we reveal the growth of silicon nanosheets where the substratesilicon interaction is minimized. Scanning tunneling microscopy measurements clearly display the atomically resolved unit cell and the small buckling of the silicene honeycomb structure. Similar to the carbon atoms in graphene, each of the silicon atoms has three nearest and six second nearest neighbors, thus demonstrating its dominant sp $^{2}$ configuration. Our scanning tunneling spectroscopy investigations confirm the metallic character of the deposited silicene, in excellent agreement with our band structure calculations that also exhibit the presence of a Dirac cone.
\end{abstract}

KEYWORDS: silicene, 2D nanomaterials, scanning tunneling microscopy, density functional theory,

ab initio molecular dynamics simulations, electronic density of states measurement and calculations, silicon growth

S ilicon-based approaches have attracted enormous attention since the first steps of nanotechnology have become a reality. ${ }^{1}$ The fruitful results obtained for graphene $e^{2,3}$ gave new momentum to the research in two-dimensional (2D) materials. ${ }^{4,5}$ Recently, silicon in a $2 \mathrm{D}$ configuration, called "silicene", has received great interest due to its compatibility with existing semiconductor technologies. Silicene consists of a honeycomb lattice of atoms akin to those in graphene. The main difference, however, resides in its structural arrangement since silicene is not atomically flat like graphene, but it shows a buckled configuration with a low, although sizable, wrinkling of the surface. ${ }^{6-8}$ Silicene may possess unique properties which provide rich opportunities for further engineering; ${ }^{9}$ for instance, an applied external electric field ${ }^{10,11}$ or interface interactions ${ }^{12,13}$ can modify its band structure, as shown in a recent proof-of-concept silicene field-effect transistor. ${ }^{14}$

Silicon crystallizes in a diamond cubic crystal structure and does not naturally form any $2 \mathrm{D}$ structures as carbon does in the form of graphite. ${ }^{15}$ Therefore, considerable research efforts have been reported for the synthesis of silicene on substrates such as silver ${ }^{16-22}$ and others ${ }^{23,24}$ that can act as a template for its formation. In spite of the large number of experimental results reported, the epitaxial growth of silicene on silver has been recently questioned. ${ }^{25-27}$ It was observed that $\mathrm{Si}$ atoms strongly interact with the Ag substrate and penetrate into the first layer of the silver surface, expelling Ag atoms and inducing reconstructions, faceting, or growing reconstructed islands inside the first layer rather than remaining on top of the substrate. This drawback can be ascribed to the significant $\mathrm{p}-\mathrm{d}$ hybridization occurring between the electronic states of the silicon layer and those of silver. ${ }^{28}$ Furthermore, the structural nature of silicene multilayers grown on $\mathrm{Ag}$ has also been severely questioned. It was revealed that the reported $(\sqrt{3} \times$ $\sqrt{ } 3) R 30^{\circ}$ surface reconstruction may actually be traced back to a layer of silver atoms rising to the surface and not to pristine $\mathrm{Si}^{29}$ These observations imply that the growth dynamics of an ultrathin silicon layer on any metal substrate is extremely complicated and does not necessarily result into genuine silicene. $^{30}$

In view of a viable solution to the above-mentioned issues associated with metallic substrates, it has been shown by firstprinciples calculations that a silicene sheet remains stable on

Received: September 13, 2016

Accepted: November 10, 2016

Published: November 10, 2016 
different inert and nonmetallic substrates. ${ }^{31-33}$ Here, following the same idea, we use a highly oriented pyrolytic graphite (HOPG) substrate that, due to its $\mathrm{sp}^{2}$ configuration, approximates well the electronic and structural properties of a fully honeycombed structure and provides chemical inertness. Hence, we show that the deposition of silicon atoms produces well-defined discrete areas on the HOPG substrate with a honeycomb structure with a lattice parameter of about $0.4 \mathrm{~nm}$, which is expected to be typical for genuine free-standing silicene layer, with negligible interaction with the supporting substrate. Similar to the $\mathrm{C}$ atoms in graphene, each of those $\mathrm{Si}$ atoms only has three nearest neighbor atoms for a true silicene arrangement.

We have performed a combined experimental and theoretical study providing strong evidence that $2 \mathrm{D}$ alloy-free silicene can be grown on top of HOPG. X-ray photoemission spectroscopy (XPS) helps to investigate the composition of the grown silicon layer on a large scale, while atomic force microscopy (AFM) gives evidence for the formation of large nanosheets and small Si 3D clusters on the substrate. Scanning tunneling microscopy (STM) measurements show our silicene unit cell and reveal the small buckling. Our density functional theory (DFT) calculations nicely agree with the structural results obtained experimentally. Moreover, scanning tunneling spectroscopy (STS) shows the metallic character of the silicene areas. Finally, $a b$ initio molecular dynamics (AIMD) simulations explore the thermal stability of the bidimensional structure at two different temperatures (room temperature (RT) and $350{ }^{\circ} \mathrm{C}$ ) and investigate the growth mechanism of silicene on the graphite substrate.

\section{RESULTS AND DISCUSSION}

Figure 1a shows the behavior of the Si 2p and Si 2s XPS core levels obtained after deposition of one monolayer (ML) of silicon at RT (see Methods). We note that both Si peaks are highly symmetric and do not show any additional components due to oxidation processes (located between 101 and $103.5 \mathrm{eV}$ ) or due to carburization reactions with the substrate to form $\mathrm{Si}_{(1-x)} \mathrm{C}_{(x)}$ compounds. ${ }^{34,35}$ This ensures the low reactivity of the $\mathrm{Si}$ deposition at RT with the graphite underneath. The AFM image of the HOPG sample in Figure $1 \mathrm{~b}$ shows a perfectly flat and clean bare surface with well-resolved monatomic steps. Figure 1c,d (as an enlarged view of Figure 1c) shows the AFM images collected on the same substrate after $1 \mathrm{ML}$ deposition of Si maintaining the substrate at RT. As reported in Figure $1 \mathrm{~d}$, the silicene ultrathin film appears organized in a quasi-continuous layer with large patchworks (clearest regions indicated by light blue arrows) extending over tens of nanometers but not completely covering the HOPG substrate (darkest zones as indicated by white arrows) probably because of the large mismatch between the silicon and graphite lattices. In these conditions, we estimate that the percentage of coverage of HOPG is about $80 \%$. Naturally, we cannot exclude the formation of a silicon oxide layer, although a low reactivity of the silicene surface with $\mathrm{O}_{2}$ has been recently reported. ${ }^{36}$ Moreover, we note from the AFM image that the silicene areas are flat surfaces (with a roughness of $0.1-0.2 \mathrm{~nm}$ measured by the line profile of the clearest zones of Figure 1d). The white spots are due to the formation of small Si clusters ( $1 \mathrm{~nm}$ high) which decorate the HOPG monatomic steps, as indicated in (c) by white arrows.

Figure 2a,b shows typical STM images obtained after one ML deposition of silicon on HOPG kept at RT (see Methods).
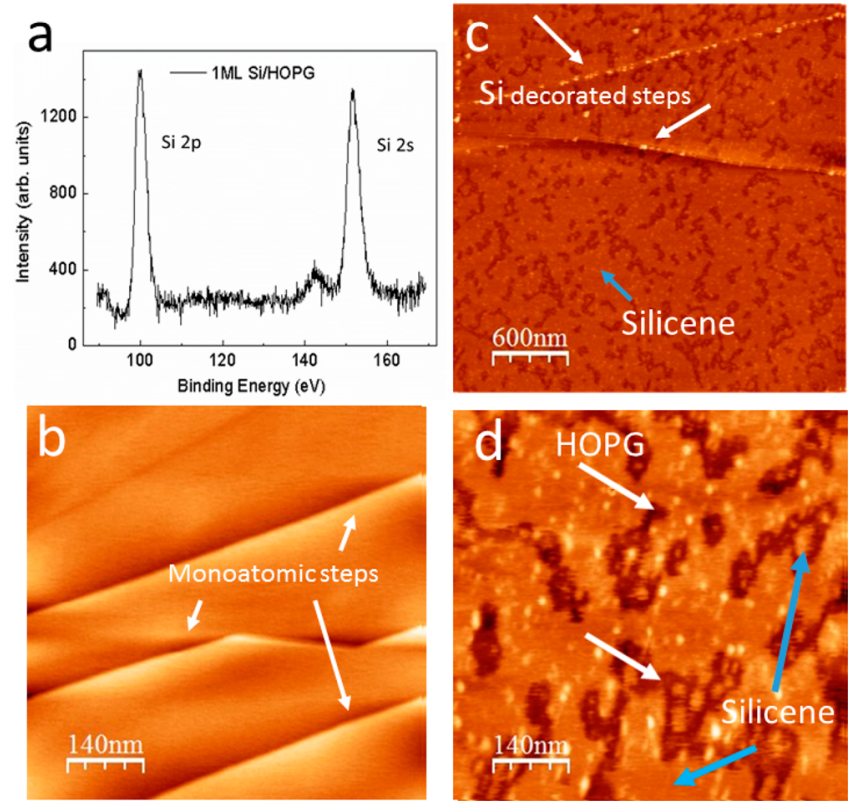

Figure 1. XPS Si $2 p$ and $2 s$ characterization and atomic force microscopy images. (a) Si $2 p$ and Si $2 s$ XPS core levels obtained after the deposition of one monolayer of silicon on HOPG at RT. We note that both $\mathrm{Si}$ components are highly symmetric and do not show any additional peaks due to oxidation or due to carburization reactions to form $\mathrm{Si}_{(1-x)} \mathrm{C}_{(x)}$ compounds. ${ }^{35,34}$ (b) AFM image of HOPG showing a flat and clean surface with several monatomic steps (whites arrows). (c) Silicon monolayer deposited at RT on HOPG. The image has been collected in air. The $\mathrm{Si}$ thin film (clearest regions indicated by a light blue arrow) appears to not completely cover the entire HOPG substrate (darkest regions) probably because of the large mismatch between the silicon and graphite atomic lattices. The white spots are very small $3 \mathrm{D} \mathrm{Si}$ clusters (1 nm height) which decorate the HOPG steps (white arrows). (d) Enlarged image of a region of the AFM image shown in (c), where the HOPG regions (white arrows), silicene patchworks (light blue arrows), and Si clusters (white spots) are shown.

We observe the formation of some small Si islands (very bright regions) rising above flatter zones. These flatter areas appear like a patchwork of hexagonal patterned regions with different lattice spacing. Their extension and size prevent their interpretation in terms of Moire interference or other artifacts induced on the HOPG surface by the silicon deposition. White circles in Figure $2 \mathrm{a}$ evidence the areas with wider lattice spacing, while in the top-left corner, a hexagonal region with a lower lattice spacing is clearly visible. In Figure $2 \mathrm{c}$,d, we report the bidimensional Fourier transform of the two STM images. Both of them confirm the STM image texture observation, exhibiting the presence of two hexagonal arrangements rotated by $30^{\circ}$ with respect to another. The external observed spots correspond to four $\{10\}$ and two $\{-11\}$ spots of HOPG. Indeed, since the distance $d_{10}$ (or equivalently $d_{-11}$ ) is equal to $\frac{\sqrt{3}}{2} a$, where $a$ is the hexagonal lattice parameter, by measuring the distance between the spots and the center, we obtained a value of $a=0.24 \pm 0.01 \mathrm{~nm}$ that corresponds well to the lattice constant of carbon atoms arranged in a hexagonal pattern for a bare HOPG surface. The inner spots correspond to the atoms arranged with a wider lattice parameter. The ratio between a couple of outer and inner spots is $1.71 \pm 0.01$, which is a factor of $\sqrt{3}$ of the HOPG hexagonal lattice parameter and 


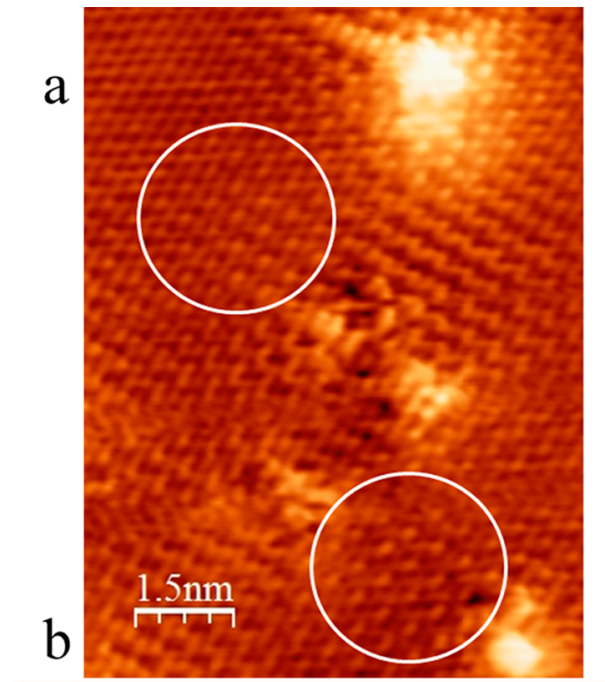

C
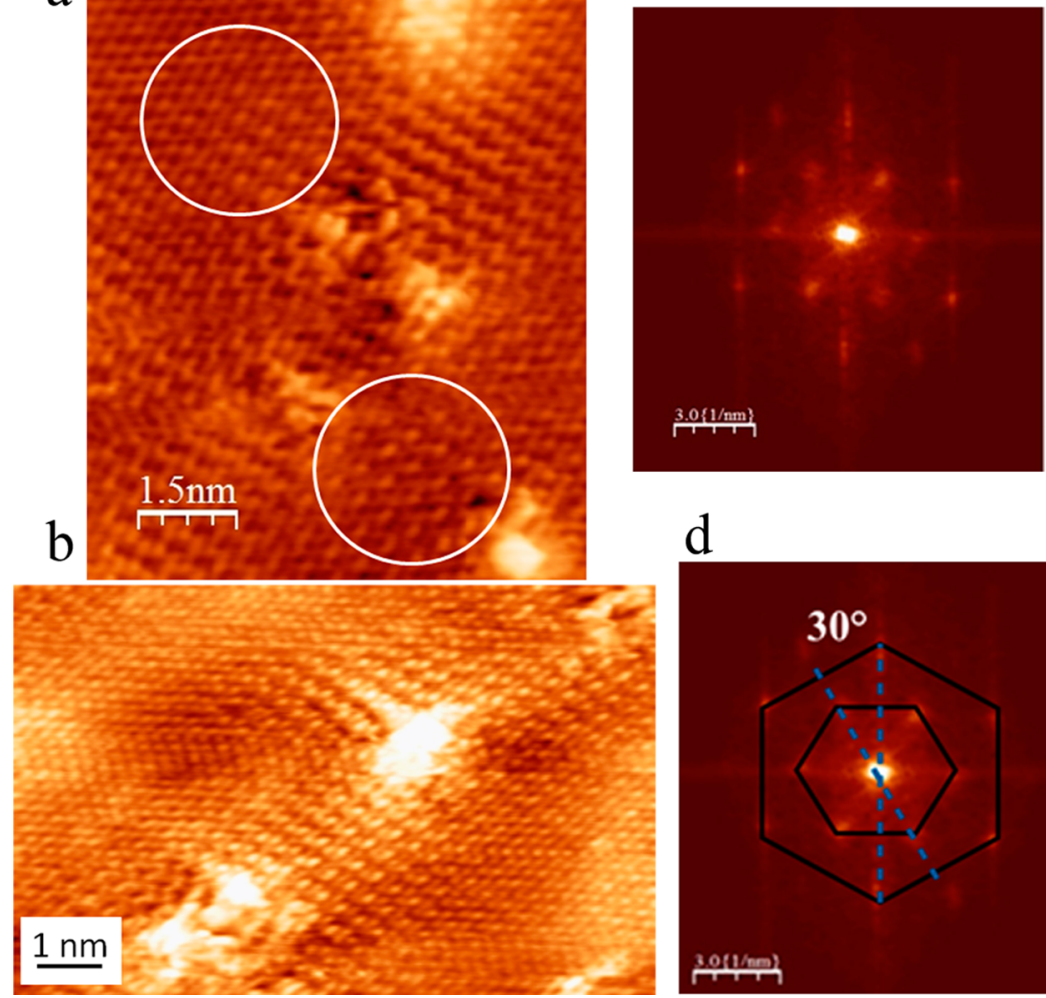

d

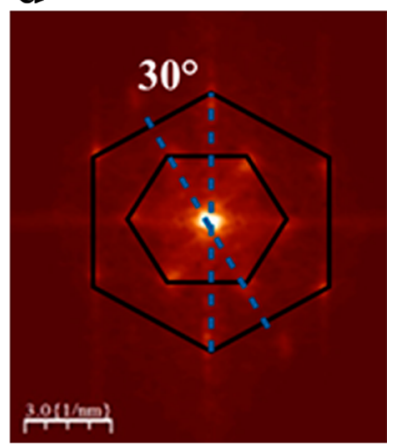

Figure 2. Scanning tunneling microscopy images of one monolayer silicon deposited on HOPG at room temperature. (a,b) STM images (collected with $V_{\text {sample }}=+0.3 \mathrm{~V}$, tunneling current, $I_{\text {tunn }}=0.3 \mathrm{nA}$ ) after one silicon monolayer deposited on a HOPG substrate at RT. Note the coexistence of silicene areas and small, $1 \mathrm{~nm}$ high, silicon clusters (white regions). (c,d) Bidimensional Fourier transform of the two STM images: two hexagons (the external due to HOPG and the internal due to silicene) can be noted to be rotated by about $30^{\circ}$ with respect to the other.
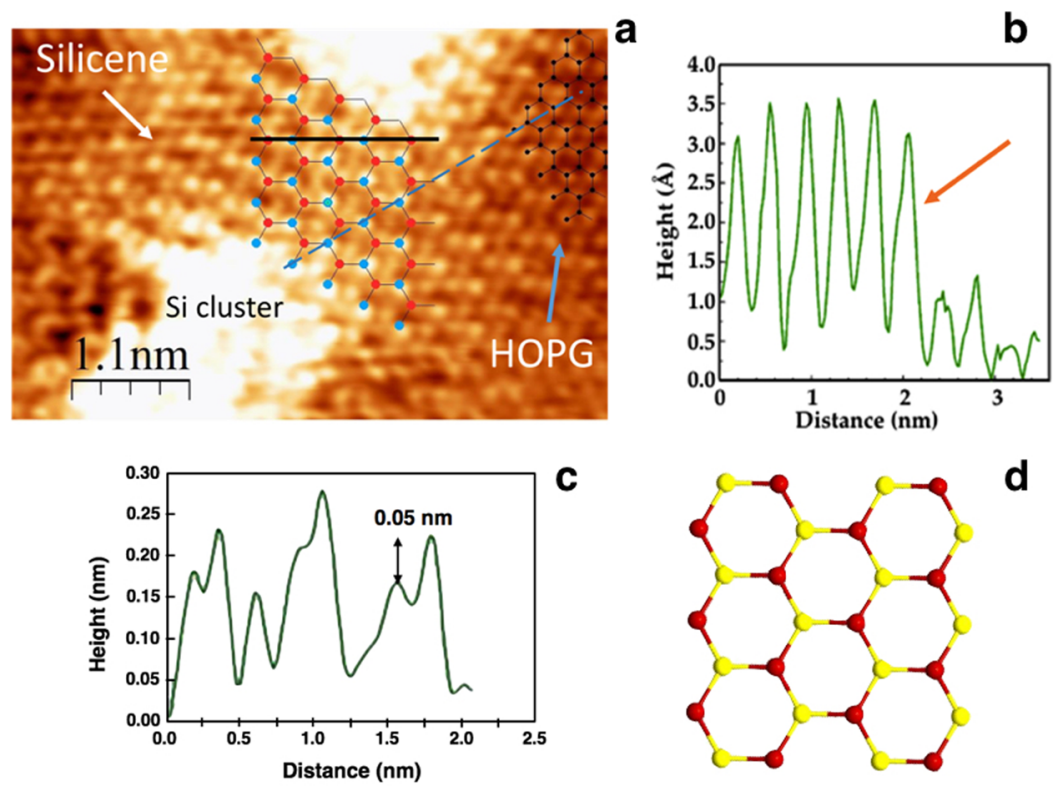

Figure 3. Scanning tunneling microscopy image of one monolayer of Si deposited on HOPG at room temperature. (a) STM image (collected with $V_{\text {sample }}=+0.3 \mathrm{~V}, I_{\text {tunn }}=0.3 \mathrm{nA}$ ) obtained with the HOPG substrate kept at RT during silicon evaporation. Ball-and-stick models have been superimposed on the image representing $\mathrm{Si}$ atoms (blue and red) and $\mathrm{C}$ atoms (black) for better understanding. (b) Line profile obtained along the blue dashed line drawn in (a). The red arrow indicates the height of the silicene layer with respect to the HOPG surface. (c) Line profile obtained along the solid black line drawn in (a) determines the presence of a buckling of about $0.05 \mathrm{~nm}$ of the silicon nearest neighbors. (d) Ball-and-stick model drawn for silicene with a low buckling of the honeycomb structure. Red balls represent the highest Si atoms, while the yellow ones are slightly lower in height. 
corresponds to a lattice parameter of $0.41 \pm 0.02 \mathrm{~nm}$. This is strong evidence in favor of the formation of a $(\sqrt{3} \times \sqrt{ } 3) R 30^{\circ}$ $\mathrm{Si}$ atom adlayer. To support this finding, in the Supporting Information, we show in Figure 1a-SI the STM image and in Figure $1 \mathrm{~b}$-SI the corresponding Fourier transform of the bare HOPG before Si deposition. It is worth noting that the Fourier transform presents only a single hexagonal pattern, giving a lattice parameter of $0.24 \pm 0.01 \mathrm{~nm}$. Interestingly, the Si adlayer hexagonal pattern of Figure 2 is different from that of the HOPG (see also the discussion of Figure 3 below), in which only one atom out of two of the hexagonal honeycomb structure is observed (note for comparison Figure $1 \mathrm{~b}-\mathrm{SI}$ and the HOPG atomic model in Figure 1c-SI). For HOPG, this is due to the characteristic alternation between filled and empty $\mathrm{p}_{z}$ orbitals normal to the surface, while for the $\mathrm{Si}$ adlayer, all $\mathrm{Si}$ atoms can be simultaneously observed but at different heights, suggesting that the adlayer is not completely flat as for HOPG and presents a small buckling. In Figure $3 \mathrm{a}$, we show the enlarged image of the central part of Figure $2 b$, highlighting the honeycomb lattice of the Si adlayer in the region between two clusters. A ball-and-stick model representing the $\mathrm{Si}$ atom arrangement, with higher atoms in blue and lower ones in red, clearly shows a rotation of the lattice structure of the $\mathrm{Si}$ adlayer with respect to that of graphite. The experimental height of the $\mathrm{Si}$ honeycomb regions above the HOPG (indicated by a red arrow on the line profile shown in Figure $3 b$ ) is perfectly comparable with that obtained with our ab initio calculations (see below). Moreover, following the horizontal line profile drawn in Figure 3a, we identify the presence of the buckling of the silicon nearest neighbors, which results in about $0.05 \pm 0.01$ $\mathrm{nm}$. This accounts well for the theoretical model of silicene in the low buckling configuration (as discussed below) and reported in literature for the free-standing silicene ${ }^{6}$ and for silicene on graphene. ${ }^{33}$ Besides the well-known growth of $\mathrm{Si}$ clusters $^{37}$ on HOPG and on epitaxial graphene on $6 \mathrm{H}$ $\mathrm{SiC}(0001),{ }^{38}$ an observation of genuine silicene regions has been reported in the present study. The most striking difference with the existing literature on silicene growth on metallic substrates is the absence of any atomic surface reconstruction and/or superstructure. ${ }^{16,29}$

In Figure 4, we show the current versus voltage $(I-V)$ curve obtained on the silicon honeycomb region. The experimental curve in Figure 4a is an average of many acquisitions, where several currents and distances from the tip and the sample were used to discriminate the behavior of the silicene patches from the metallic character of the HOPG. It can be noticed that the silicene region reveals a metallic behavior. Figure $4 \mathrm{~b}$ reports the normalized conductance that can be related to the local density of states (LDOS), and it reveals a finite density at $E_{\mathrm{F}}$. We observe a minimum zone of states close to the Fermi level, which can be associated with the gap opening at the $\Gamma$ point for silicene and at the $\mathrm{K}$ point for HOPG of the silicene/HOPG system. This result nicely agrees with the computed LDOS from the energy band structure (as discussed in the theoretical section) and reported in the same figure as a red dotted line.

Finally, we would like to point out an inherent problem with our recording of the STM images: at present, we have to operate our STM at room temperature. From our AIMD simulations (see below), we know that silicene atoms that are bonded with van der Waals forces to the HOPG substrate experience much larger displacements around their equilibrium position than silicon atoms that are bonded covalently to a silver substrate. In order to achieve the same image quality as

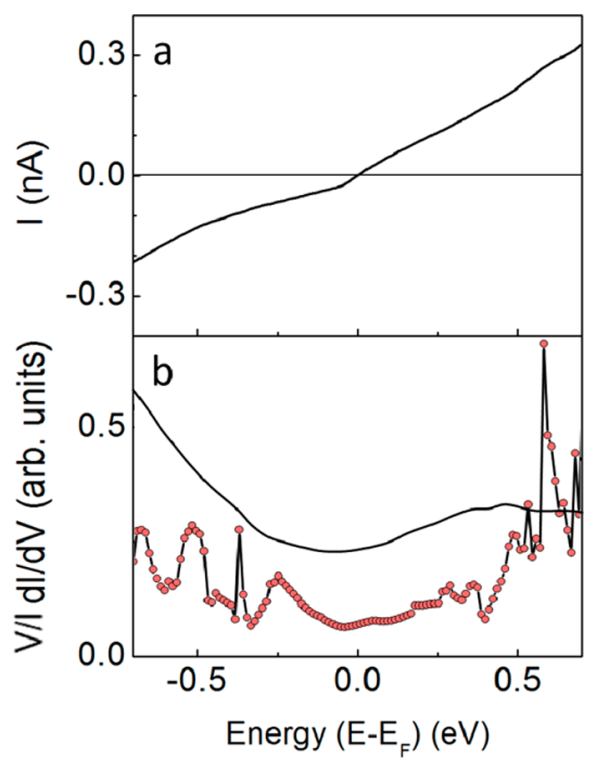

Figure 4. Scanning tunneling spectroscopy measurement. (a) $I-V$ curve registered on silicene on HOPG. We observe a straight line around the Fermi level (located at $0.0 \mathrm{eV}$ ) showing the metallic character, i.e., ohmic behavior. (b) Normalized conductance calculated from curve (a) related to the local density of states further confirming the metallic character of the obtained silicene. Red dotted line is the theoretical density of states computed through the energy band structure of the silicene/HOPG system as discussed below.

those previously published on silver, we estimate that we have to cool our samples to about liquid nitrogen temperatures to reduce the large root-mean-square displacement.

First-principle calculations were performed in the framework of DFT (see Methods) to address the geometry and structural stability of a silicene monolayer on a HOPG surface. The above STM images have shown different rotation angles between the silicene and graphite lattices. Due to the weak van der Waals forces holding the silicene to the graphite surface, one can actually imagine that the silicene layer is "floating" quite freely on top of the HOPG surface and, therefore, can assume different configurations for different silicene areas even on the same sample.

The calculations for the relaxed silicene on a graphite structure with $30^{\circ}$ rotation angle between the two hexagonal patterns, depicted in Figure 5a, yields a $0.051 \mathrm{~nm}$ buckling of the silicene layer, and its distance to the top layer of the graphite surface is $0.333 \mathrm{~nm}$. The silicene bond length is 0.225 $\mathrm{nm}$, and its bond angle is around $114^{\circ}$. The lattice parameters of silicene and graphite are 0.379 and $0.246 \mathrm{~nm}$, respectively, in excellent agreement with our STM measurements obtained for RT deposition.

Figure $5 \mathrm{~b}$ shows the calculated band structure of silicene on HOPG for a silicene area with about $10^{\circ}$ rotation angle between the two hexagonal patterns in the $\mathrm{M}-\mathrm{K}, \mathrm{K}-\Gamma$, and $\Gamma-$ $M$ directions, revealing one Dirac cone at the $\Gamma$ point belonging to silicene. We stress that in the free-standing case, the Dirac cone of silicene is at the $\mathrm{K}$ point as well as the Dirac cone of bare graphene. ${ }^{6}$ When the silicene-graphene system is built, the Brillouin zones of both graphene and silicene are rotated with respect to the other. Hence, the $\mathrm{K}$ point of silicene falls onto the $\Gamma$ point of the Brillouin zone of the silicene-graphene system. Our band structure is quite similar to the band 

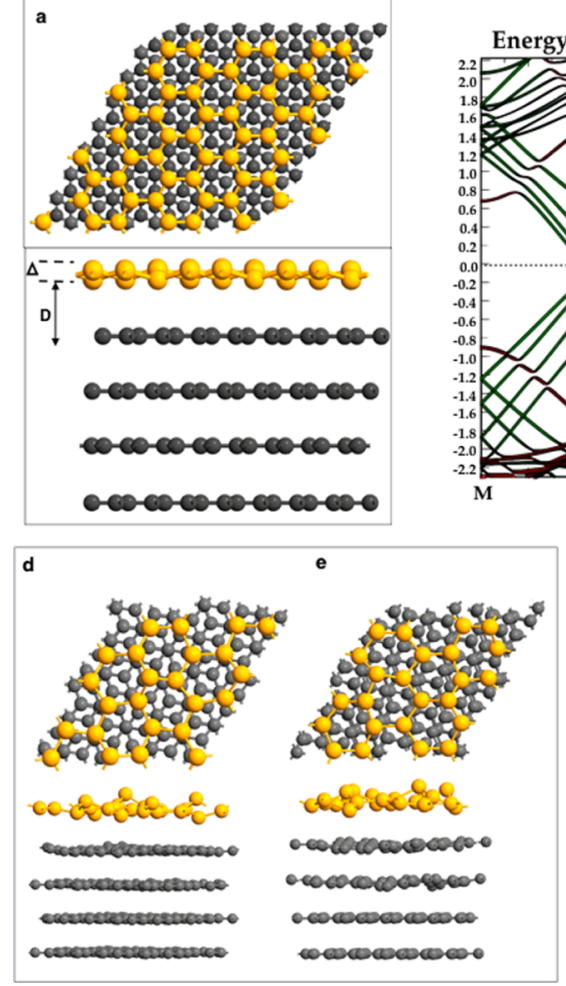

Silicene/HOPG Band Structures
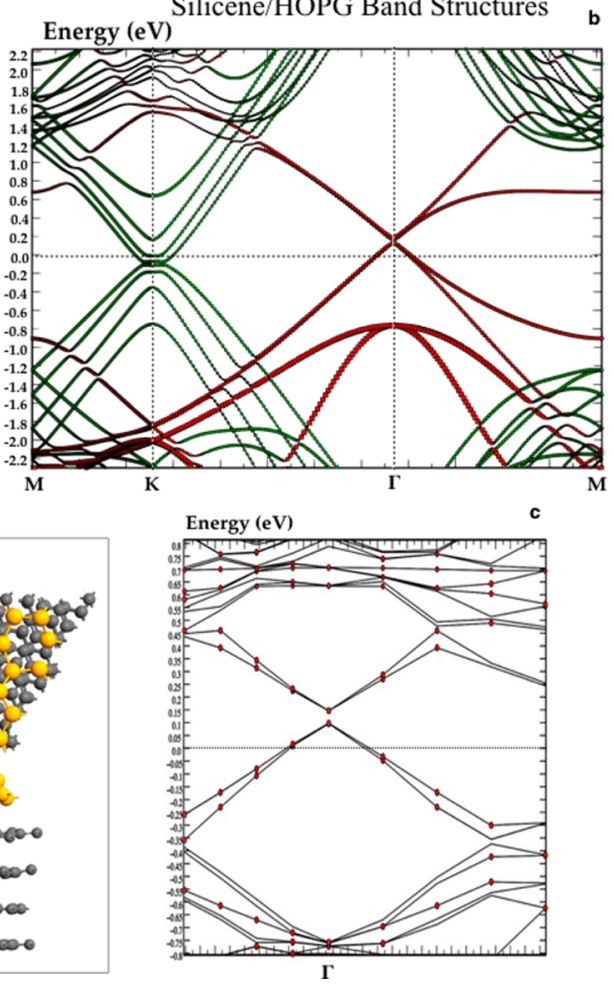

Figure 5. Atomic structure model, band structure, and AIMD snapshots of silicene on HOPG. (a) Top and side views of the optimized structure obtained by DFT calculations which show the silicene layer at a distance $(D)$ of $0.333 \mathrm{~nm}$ above the graphite surface with a buckling parameter $(\Delta)$ of $0.051 \mathrm{~nm}$. Silicon atoms are shown in yellow and carbon atoms in gray. (b) Band structures of silicene on graphite with a $10^{\circ}$ rotation angle between the two hexagonal patterns; projected bands of $\mathrm{Si}$ and $\mathrm{C}$ are highlighted in red and green, respectively. (c) Band structures of silicene on graphite with a $30^{\circ}$ rotation angle between the two hexagonal patterns highlighting the Dirac cone of silicene at the $\Gamma$ point. (d,e) Snapshots from AIMD simulations showing the structure (top and side views) obtained at RT (d) and at $350{ }^{\circ} \mathrm{C}(\mathrm{e})$, revealing the stability of the silicene layer well above RT.

structure of silicene on graphene, as calculated by Cai et al. ${ }^{33}$ The latter also showed that the $\mathrm{p}_{z}$ states of $\mathrm{Si}$ are responsible for the $\pi$ bonds in the vicinity of the Fermi level and that the Dirac cone of silicene is mapped to $\Gamma$ while the Dirac point of graphene stays at the $\mathrm{K}$ point. Furthermore, the calculated Dirac cone of silicene on HOPG is somewhat shifted above the Fermi energy, which can be attributed to a slight charge transfer between silicene and its substrate. Free-standing silicene is predicted to have a band gap of $1.55 \mathrm{meV} .{ }^{39}$ When silicene is formed on graphite, we find that this gap increases to about 37 $\mathrm{meV}$; that is, it is quite comparable to the silicene band gap on graphene of $57 \mathrm{meV} .{ }^{33}$ Consequently, we can conclude that increasing the number of graphene layers in our graphite surface model would not affect the band gap in the silicene sheet and that the small interaction occurs only between the silicene sheet and the top graphite layer. This energy gap is induced by the substrate, which partially breaks the sublattice symmetry of silicene due to the inhomogeneous van der Waals interaction. The band structure of silicene on HOPG with a $30^{\circ}$ rotation angle between the two hexagonal patterns in Figure $5 c$ shows the Dirac cone of silicene at the $\Gamma$ point, slightly shifted above the Fermi level with a band gap of about $48 \mathrm{meV}$.

To demonstrate the stability of the honeycomb structure for the silicene on HOPG structure, we have performed AIMD simulations. A $(2 \times 2)$ supercell model of a silicene monolayer on HOPG was at first fully optimized with standard $a b$ initio methods. Then, we carried out our AIMD simulations at RT and at $350{ }^{\circ} \mathrm{C}$ for more than 3 ps. Our simulations reveal that the silicene layer remains stable even at temperatures well above RT (see Figure 5d,e). While the in-plane hexagonal lattice structure of silicene is preserved for both temperatures, we find that the buckling of the silicene sheet slightly increases with temperature. To investigate the stability of silicene at elevated temperature for some time in experiments, we carried out AIMD simulations. Here, we would like to point out that the time scale in the AIMD simulation method is on the order of picoseconds, which is too short compared to the time scale in experiments; therefore, we have chosen to study the stability of the system at extremely high temperatures. To this end, we heated the silicene on the graphite system from 25 to $725{ }^{\circ} \mathrm{C}$ during $5.6 \mathrm{ps}$, and then we applied a thermostat to keep the temperature of the system stable around $725^{\circ} \mathrm{C}$ for $4.58 \mathrm{ps}$. We noticed that the silicene structure did not break apart and convert to a $3 \mathrm{D}$ structure, but $\mathrm{Si}$ atoms remained intact in the honeycomb lattice. The relatively high stability at this extreme temperature suggests that our system should remain stable over relatively long times at less elevated temperatures.

The thermal stability of the silicene monolayer even above RT underlines the essential contribution of the van der Waals forces binding the silicon atoms in a honeycomb pattern to the graphite surface. From our simulations of a perfect silicene sheet on top of HOPG, we did not perceive any 3D silicon cluster formation even at $350{ }^{\circ} \mathrm{C}$. Therefore, we will take a closer look at the details of the growth dynamics below. At this point, however, we can already conclude that a perfect silicene sheet remains stable at such a high temperature of the substrate, supporting the STM measurements where silicene zones were observed in many regions. 
In order to go deeper in the atomic-scale view of the growth process of the silicene layer on HOPG, we have carried out AIMD simulations under realistic experimental conditions (see Methods). In Figure 6, we present the general trends observed
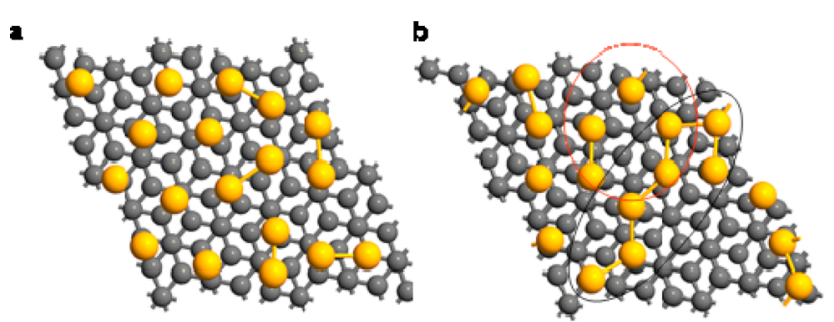

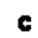
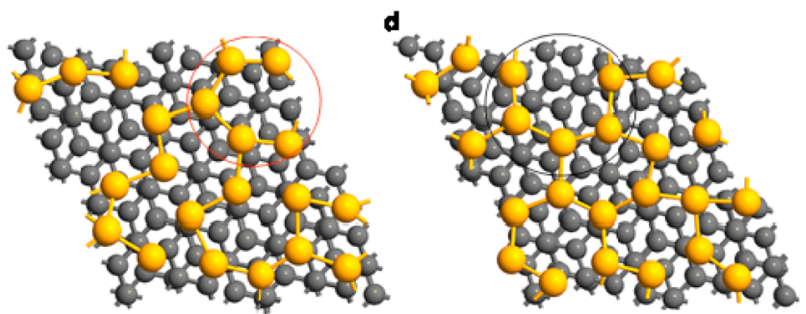

-

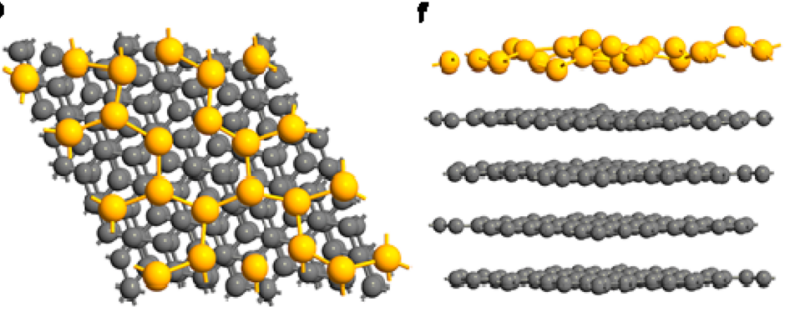

Figure 6. Snapshots from AIMD simulations of the epitaxial growth of a silicene monolayer on HOPG at RT. (a) Initial configuration: $0.75 \mathrm{ML}$ coverage of $\mathrm{Si}$ atoms randomly distributed above the graphite surface. Silicon atoms are shown in yellow and carbon atoms in gray. (b) Structure after 0.9 ps of self-organization: one hexagon of $\mathrm{Si}$ atoms starts to form. (c) Structure with $0.88 \mathrm{ML}$ coverage of $\mathrm{Si}$ atoms after 1.9 ps clearly showing two hexagons forming. (d) Structure with $0.96 \mathrm{ML}$ coverage of $\mathrm{Si}$ after $3.3 \mathrm{ps}$. (e) Structure with 1 ML coverage of Si after 3.9 ps showing that the silicene region formed with only few defects. (f) Side view of (e) underlining the flatness of the silicene sheet at RT.

during the growth. The initial structure consists of a $0.75 \mathrm{ML}$ of $\mathrm{Si}$ atoms, that is, $18 \mathrm{Si}$ atoms, randomly distributed at about $0.35 \mathrm{~nm}$ above the graphite surface with relatively large lateral distances between them, such that the initial interaction between those $\mathrm{Si}$ atoms before moving was negligible, as depicted in Figure 5a. Following the trajectories for 900 molecular dynamics (MD) steps (0.9 ps evolution time), we can notice from Figure $5 \mathrm{~b}$ how $\mathrm{Si}$ atoms bind together in a long chain (black ellipse), while other $\mathrm{Si}$ atoms move closer to each other to form the first hexagon (red circle). After 1 ps of system evolution from the starting configuration, three $\mathrm{Si}$ atoms are added on the bare regions of the graphite surface to have a 0.88 ML coverage. We notice that two hexagons are formed (red circles) along with small polygonal rings after $900 \mathrm{MD}$ steps (Figure 5c). After another $1 \mathrm{ps}$, three more Si atoms are added to get a $0.96 \mathrm{ML}$ coverage. Figure $5 \mathrm{~d}$ is a snapshot of the structure resulting from its self-organization after about 1300 MD steps. Nearly all $\mathrm{Si}$ atoms in the system now bind in a hexagonal network with one large polygon (black ellipse).
Finally, after around 1.4 ps of system evolution, one more $\mathrm{Si}$ atom is added in a random location to reach a full coverage ( 1 $\mathrm{ML})$. Figure $5 \mathrm{e}$ is a snapshot of the system trajectory after an additional $633 \mathrm{MD}$ steps showing the honeycomb network of the silicene monolayer with only little defects. The formed silicene sheet lies about $0.4 \mathrm{~nm}$ above the HOPG surface, which agrees very well with our STM measurements.

Two different growth mechanisms are unveiled in the AIMD simulations: a two-dimensional one where a silicene sheet was observed and a three-dimensional one where 3D silicon islands were formed. We can explain these growth mechanisms as follows: the lower the temperature of the graphite surface, the more perfectly a $2 \mathrm{D}$ plane based on van der Waals forces is defined. If the temperature gets so high that the out-of-plane vibrational amplitude of the carbon atoms gets anywhere close to half of the $\mathrm{Si}-\mathrm{Si}$ interatomic distance, the van der Waals potential will no longer define a plane, but rather a volume, favoring the onset for the formation of $3 \mathrm{D} \mathrm{Si}$ clusters. To confirm this hypothesis, we checked the maximum vibrational amplitude of carbon atoms in the top layer of the graphite substrate and found it to be around 0.04 and $0.10 \mathrm{~nm}$ for RT and $350{ }^{\circ} \mathrm{C}$, respectively. Consequently, we propose tentatively that perfect $2 \mathrm{D}$ silicene layers cannot be created on $\mathrm{HOPG}$ at substrate temperatures exceeding well above $350{ }^{\circ} \mathrm{C}$. Moreover, we have carried AIMD simulations at lower temperatures. Here, we found that by decreasing the temperature, even down to $-225{ }^{\circ} \mathrm{C}$, we obtain a spontaneous organization of silicon atoms above the HOPG surface. However, for all temperatures below RT, the formation of hexagons was accompanied by other polygons such as pentagons and heptagons. Therefore, we suggest tentatively that a temperature of $25^{\circ} \mathrm{C}$ presents the low-temperature limit for the formation of a default-free honeycomb structure.

Interestingly, we have observed at an atomistic scale how $\mathrm{Si}$ hexagons spontaneously form on top of the HOPG substrate at RT. This growth mechanism only becomes possible due to the van der Waals interactions between silicon and carbon atoms which are strong enough to define a flat growth plane necessary for the silicene sheet formation but weak enough to leave the silicene properties largely unchanged from the free-standing silicene case; that is, in the present system, one can create a stable silicene monolayer without any covalent bonds to its supporting substrate underneath.

\section{CONCLUSIONS}

Several silicene areas with a structure very similar to that of graphene have been obtained by the deposition of one monolayer of silicon on an inert HOPG substrate at RT and under ultrahigh vacuum (UHV) conditions. AFM images support the formation of a quasi-continuous $2 \mathrm{D}$ silicon layer leaving some areas of HOPG uncovered together with the formation of small 3D Si clusters. STM data support the existence of a two-dimensional graphene-like structure with a very small buckling $(0.05 \mathrm{~nm})$ of the silicon nearest neighbors. The atomic resolution images obtained by STM do not show any surface reconstruction, as found when silicene is deposited on metal surfaces, suggesting a negligible electronic interaction/ hybridization with the substrate underneath. This observation suggests that a growth mode with a full release of strain occurs.

AIMD simulations predict the thermal stability of a perfect silicene monolayer on a HOPG surface at RT and $350{ }^{\circ} \mathrm{C}$, highlighting the essential role of van der Waals forces bonding silicene to the surface. Simulations of the growth mechanism of 
the silicene sheet on HOPG at RT show how silicon hexagons spontaneously form and remain at a vertical distance corresponding to the van der Waals distance above the surface.

HOPG is a good candidate to form silicene layers with remarkable electronic properties very different from any other known silicon-based nanostructures. The growth of silicene on a nonmetallic and inert substrate is an important step toward the realization of alloy-free silicene layers with properties that approach the ones of free-standing silicene. In comparison to graphene, silicene offers the prospect of full compatibility with existing silicon technologies. We believe that we succeeded in observing these small, but true silicene areas because of a rather slow deposition rate of silicon, and we are confident that in the future by using more sophisticated growth methods (as molecular beam epitaxy) larger silicene areas can be obtained. Only in the case of continuous silicene layer formation, we will be able to detach and transfer this ultrathin film on a device as already done for silicene grown on $\operatorname{Ag}(111) .{ }^{14}$ In future works, the ideal deposition temperature has, for instance, to be determined more carefully: hot enough to overcome all energy barriers to silicene growth but low enough to avoid 3D cluster formation. Optimized experimental deposition conditions will favor the extent of the pure silicene domains, eventually pushing them to the size limits imposed by the terrace size of the graphite substrate possibly making the use of graphene instead of HOPG necessary for extremely large silicene layers. The ultimate silicene/graphene heterostructure can then be lifted off from the underlying substrate and deposited on $\mathrm{SiO}_{2}$ as already reported in the literature. The silicene/graphene/ $\mathrm{SiO}_{2}$ system can then be integrated in functional devices. Such systems can be grown on a very large scale corresponding to the requirements of the electronic industry. Besides this immediate compatibility with powerful silicon technologies and the absence of any alloying problems, the silicene on graphite system presents a much less expensive solution than strategies based on silver substrates. Therefore, the present research is in the forefront of $2 \mathrm{D}$ materials with revolutionary future applications for nanotechnological and optoelectronic devices as, for example, ultra-high-speed electronic devices, high-efficiency photovoltaic solar cells, and white-light-emitting diodes. ${ }^{40}$

\section{METHODS}

Experimental. A HOPG (from GE Advanced Ceramics, USA, 12 $\mathrm{mm} \times 12 \mathrm{~mm} \times 1 \mathrm{~mm}$ ) sample was used as a substrate. A fresh surface of graphite was obtained by peeling the HOPG substrate with scotch tape in a nitrogen atmosphere and was then transferred into a UHV chamber.

High-purity silicon (Sil'tronix ST, $\rho=1-10 \Omega \cdot \mathrm{cm}$ ) was evaporated from a tungsten basket-shaped crucible located at $200 \mathrm{~mm}$ from the substrate. The deposition was done in UHV conditions (base pressure low $10^{-10}$ Torr) and at a constant rate of $0.01 \mathrm{~nm} / \mathrm{min}(0.04 \mathrm{ML} /$ $\mathrm{min}$ ) monitored by an Inficon quartz balance (properly calibrated for the Si density and carefully positioned close to the sample holder). Deposition was carried out keeping the substrate at RT. Because of the peculiar nature of the HOPG substrate, which is formed by large and highly flat single crystals disoriented in the basal plane, with respect to the other around the (0001) growth axis, it was not possible to perform reliable low-energy electron diffraction investigations. The diffraction pattern, in fact, has formed by a series of continuous circles with some spots revealing the crystalline structure of each graphite crystal.

Samples were studied in situ at RT by XPS, STM, and STS. C 1s and $\mathrm{Si} 2 \mathrm{p}$ and $\mathrm{Si} 2 \mathrm{~s}$ XPS photoemission spectra were measured in situ using a non-monochromatic $\mathrm{Al} \mathrm{K} \alpha$ radiation $(1486.6 \mathrm{eV})$ and a double-pass CMA operating in retarding mode. The energy scale was calibrated with reference to the binding energy of the $S i 2 p_{3 / 2}$ peak from a clean $\mathrm{Si}(100) 2 \times 1$ reconstructed sample and measured to be $99.5 \pm 0.9 \mathrm{eV}$ with respect to the Fermi level. STM imaging was performed using an Omicron-STM system with electrochemically etched tungsten tips. The STM was calibrated by acquiring atomically resolved images of the bare HOPG. All images were acquired in the constant mode and were unfiltered apart from the rigid plane subtraction. The STS measurements helped us to investigate the local electron density of states near the Fermi level. During the acquisition, the tunneling current was registered as a function of the applied bias ( $I$ $v s V$ ), the feedback loop was disabled, and the set-point current, which regulates the tip-sample distance, remained unchanged during the voltage scan. Current-voltage curves were collected over grids of points equally spaced on the scanned sample area, and the $I-V$ spectra were averaged over a set of several curves. AFM measurements were performed in air, on the same sample used for STM measurements, with a XE-100 of PSIA apparatus with sharp silicon tips from advanced scanning probe microscopy.

Calculations. Ab initio calculations were performed by using DFT within the generalized gradient approximation including van der Waals corrections as implemented in the Vienna ab initio simulation package (VASP). ${ }^{41,42}$ The Perdew-Burke-Ernzerhof ${ }^{43}$ with van der Waals-D2 functional ${ }^{44}$ was used to describe the exchange-correlation interaction. The core electrons were described by the projector-augmented wave method. ${ }^{45}$ The plane-wave basis set was restricted to a cutoff energy of $400 \mathrm{eV}$. The HOPG surface was modeled by a four-layer slab in an $\mathrm{ABAB}$ stacking of graphene within periodic boundary conditions, keeping the bottom two layers fixed. The vacuum region is as thick as $2.0 \mathrm{~nm}$. The entire system, silicene on top of graphite, was fully relaxed by a conjugate gradient method until the forces acting on each atom were less than $0.1 \mathrm{eV} / \mathrm{nm}$. The convergence criterion of total energy for self-consistent field calculations was chosen to be $10^{-4} \mathrm{eV}$. We have used $3 \times 3 \times 1,9 \times 9 \times 1$, and $11 \times 11 \times 1$ k-point meshes including the $\Gamma$ point in order to sample the supercells with 30,10 , and $20^{\circ}$ rotation angles between the two hexagonal patterns, respectively. $A b$ initio molecular dynamics simulations within the NVT ensemble, ${ }^{46}$ using the Nose-Hoover thermostat ${ }^{47}$ for temperature control, were performed using VASP. In order to assess the thermal stability of the silicene sheet, a larger model of the silicene on graphite system was constructed. Notably, a $(2 \times 2)$ supercell (i.e., 2 times the length of the optimized silicene on the graphite system, with $10^{\circ}$ rotation angle between the two hexagonal patterns in both $x$ - and $y$-directions) with dimensions of $1.3 \mathrm{~nm} \times 1.3 \mathrm{~nm}$ was exploited. A similar graphite model with the same dimensions was used to study the growth mechanism of a silicene sheet. The time step for all AIMD simulations was $1.0 \mathrm{fs}$.

A four-layer slab, with 56 carbon atoms per layer, modeled the graphite surface. In the experiments, $\mathrm{Si}$ atoms were evaporated at an extremely small deposition rate $(0.01 \mathrm{~nm} / \mathrm{min})$; that is, a deposited $\mathrm{Si}$ atom will have lost all of its initial impact information a long time before the next $\mathrm{Si}$ atom will arrive. Consequently, we have started the simulation of the growth mechanism with $\mathrm{Si}$ atoms randomly positioned on top of the graphite surface and not by explicitly simulating the deposition process atom by atom. It is necessary to introduce such a model because it is impossible to follow the growth with AIMD methods on a time scale of several minutes. To comply with the experimental conditions, we have started the simulations with a coverage of $\mathrm{Si}$ atoms above the graphite surface, which was less than $1 \mathrm{ML}$, followed the system evolution at RT, and then added Si atoms successively until we reached a $1 \mathrm{ML}$ coverage as in the experiment.

\section{ASSOCIATED CONTENT}

\section{S Supporting Information}

The Supporting Information is available free of charge on the ACS Publications website at DOI: 10.1021/acsnano.6b06198.

Figure 1-SI (PDF) 


\section{AUTHOR INFORMATION}

\section{Corresponding Authors}

*E-mail: decrescenzi@roma2.infn.it.

*E-mail: isabelle.berbezier@im2np.fr.

*E-mail: holger.vach@polytechnique.edu.

\section{ORCID}

\section{Maurizio De Crescenzi: 0000-0002-2935-8714}

\section{Author Contributions}

I.B. and H.V. conceived the project; M.D.C. and I.B. conceived and designed the experiments and supervised the work; M.D.C., M.S., and P.C. performed the STM and STS experiments and analyzed the results. I.B., M.A., and A.R. performed AFM experiments and discussed the results; F.J., J.P., and H.V. developed the theoretical model and made the calculations. All authors contributed to the scientific discussions, manuscript preparation, and final revision.

\section{Notes}

The authors declare no competing financial interest.

\section{ACKNOWLEDGMENTS}

M.D.C., P.C., and M.S. would like to acknowledge the European Community for the RISE Project CoExAN GA644076. H.V., F.J., and J.P. gratefully acknowledge Kostya Ostrikov for the initial fruitful discussions on silicene; the HPC centers IDRIS (Grant i2014-090642), and CERMM for computational resources; the Hariri Foundation for Sustainable Human Development for the scholarship awarded to F.J.; and the French government and the French embassy in Korea for the Blaise Pascal Scholarship given to J.P.

\section{REFERENCES}

(1) Sheka, E. F. Why $\mathrm{sp}^{2}$-Like Nanosilicons Should Not Form: Insight from Quantum Chemistry. Int. J. Quantum Chem. 2013, 113, 612-618.

(2) Novoselov, K. S.; Geim, A. K.; Morozov, S. V.; Jiang, D.; Zhang, Y.; Dubonos, S. V.; Grigorieva, I. V.; Firsov, A. A. Electric Field Effect in Atomically Thin Carbon Films. Science 2004, 306, 666-669.

(3) Berger, C.; Song, Z.; Li, T.; Li, X.; Ogbazghi, A. Y.; Feng, R.; Dai, Z.; Marchenkov, A. N.; Conrad, E. H.; First, P. N.; de Heer, W. A. Ultrathin Epitaxial Graphite: 2D Electron Gas Properties and a Route Toward Graphene-Based Nanoelectronics. J. Phys. Chem. B 2004, 108, 19912-19916.

(4) Zhu, F.; Chen, W.; Xu, Y.; Gao, C.; Guan, D.; Liu, C.; Qian, D.; Zhang, S.; Jia, J. Epitaxial Growth of Two-Dimensional Stanene. Nat. Mater. 2015, 14, 1020-1025.

(5) Davila, M. E.; Xian, L.; Cahangirov, S.; Rubio, A.; Le Lay, G. Germanene: A Novel Two-Dimensional Germanium Allotrope Akin to Graphene and Silicene. New J. Phys. 2014, 16, 095002.

(6) Cahangirov, S.; Topsakal, M.; Akturk, E.; Sahin, H.; Ciraci, S. Two- and One- Dimensional Honeycomb Structures of Silicon and Germanium. Phys. Rev. Lett. 2009, 102, 236804.

(7) Takeda, K.; Shiraishi, K. Theoretical Possibility of Stage Corrugation in $\mathrm{Si}$ and Ge Analogs of Graphite. Phys. Rev. B: Condens. Matter Mater. Phys. 1994, 50, 14916-14922.

(8) Houssa, M.; Pourtois, G.; Afanas'ev, V. V.; Stesmans, A. Can Silicon Behave Like Graphene? A First-Principles Study. Appl. Phys. Lett. 2010, 97, 112106.

(9) Jia, T.-T.; Zheng, M.-M.; Fan, X.-Y.; Su, Y.; Li, S.-J.; Liu, H.-Y.; Chen, G.; Kawazoe, Y. Band Gap On/Off Switching of Silicene Superlattice. J. Phys. Chem. C 2015, 119, 20747-20754.

(10) Ni, Z.; Liu, Q.; Tang, K.; Zheng, J.; Zhou, J.; Qin, R.; Gao, Z.; Yu, D.; Lu, J. Tunable Bandgap in Silicene and Germanene. Nano Lett. 2012, 12, 113-118.
(11) Drummond, N. D.; Zolyomi, V.; Fal'ko, V. I. Electrically Tunable Band Gap in Silicene. Phys. Rev. B: Condens. Matter Mater. Phys. 2012, 85, 075423.

(12) Lin, C.-L.; Arafune, R.; Kawahara, K.; Kanno, M.; Tsukahara, N.; Minamitani, E.; Kim, Y.; Kawai, M.; Takagi, N. Substrate-induced Symmetry Breaking in Silicene. Phys. Rev. Lett. 2013, 110, 076801-5. (13) Gao, N.; Li, J. C.; Jiang, Q. Bandgap Opening in Silicene: Effect of Substrates. Chem. Phys. Lett. 2014, 592, 222-226.

(14) Tao, L.; Cinquanta, E.; Chiappe, D.; Grazianetti, C.; Fanciulli, M.; Dubey, M.; Molle, A.; Akinwande, D. Silicene Field-Effect Transistors Operating at Room Temperature. Nat. Nanotechnol. 2015, 10, 227-231.

(15) Le Lay, G. 2D materials: Silicene Transistors. Nat. Nanotechnol. 2015, 10, 202-203.

(16) Vogt, P.; De Padova, P.; Quaresima, C.; Avila, J.; Frantzeskakis, E.; Asensio, M. C.; Resta, A.; Ealet, B.; Le Lay, G. Silicene: Compelling Experimental Evidence for Graphenelike Two-Dimensional Silicon. Phys. Rev. Lett. 2012, 108, 155501.

(17) Lin, C.-L.; Arafune, R.; Kawahara, K.; Tsukahara, N.; Minamitani, E.; Kim, Y.; Kawai, M.; Takagi, N. Structure of Silicene Grown on $\mathrm{Ag}(111)$. Appl. Phys. Express 2012, 5, 045802.

(18) Jamgotchian, H.; Colignon, Y.; Hamzaoui, N.; Ealet, B.; Hoarau, J. Y.; Aufray, B.; Biberian, J. P. Growth of Silicene Layers on Ag (111): Unexpected Effect of the Substrate Temperature. J. Phys.: Condens. Matter 2012, 24, 172001-7.

(19) Feng, B.; Ding, Z.; Meng, S.; Yao, Y.; He, X.; Cheng, P.; Chen, L.; Wu, K. Evidence of Silicene in Honeycomb Structures of Silicon on Ag (111). Nano Lett. 2012, 12, 3507-3511.

(20) Aufray, B.; Kara, A.; Vizzini, S.; Oughaddou, H.; Léandri, C.; Ealet, B.; Le Lay, G. Graphene-Like Silicon Nanoribbons on $\mathrm{Ag}(110)$ : A Possible Formation of Silicene. Appl. Phys. Lett. 2010, 96, 183102-3.

(21) De Padova, P.; Quaresima, C.; Ottaviani, C.; Sheverdyaeva, P. M.; Moras, P.; Carbone, C.; Topwal, D.; Olivieri, B.; Kara, A.; Oughaddou, H.; Aufray, B.; Le Lay, G. Evidence of Graphene-Like Electronic Signature in Silicene Nanoribbons. Appl. Phys. Lett. 2010, 96, 261905-3.

(22) De Padova, P.; Quaresima, C.; Olivieri, B.; Perfetti, P.; Le Lay, G. Strong Resistance of Silicene Nanoribbons Towards Oxidation. J. Phys. D: Appl. Phys. 2011, 44, 312001.

(23) Fleurence, A.; Friedlein, R.; Ozaki, T.; Kawai, H.; Wang, Y.; Yamada-Takamura, Y. Experimental Evidence for Silicene on Diboride Thin Films. Phys. Rev. Lett. 2012, 108, 245501.

(24) Meng, L.; Wang, Y.; Zhang, L.; Du, S.; Wu, R.; Li, L.; Zhang, Y.; Li, G.; Zhou, H.; Hofer, W. A.; Gao, H.-J. Buckled Silicene Formation on Ir (111). Nano Lett. 2013, 13, 685-690.

(25) Prevot, G.; Bernard, R.; Cruguel, H.; Borensztein, Y. Monitoring Si Growth on Ag (111) with Scanning Tunneling Microscopy Reveals that Silicene Structure Involves Silver Atoms. Appl. Phys. Lett. 2014, 105, 213106.

(26) Ronci, F.; Serrano, G.; Gori, P.; Cricenti, A.; Colonna, S. Silicon Induced Faceting at the $\mathrm{Ag}$ (110) Surface. Phys. Rev. B: Condens. Matter Mater. Phys. 2014, 89, 115437.

(27) Satta, M.; Colonna, S.; Flammini, R.; Cricenti, A.; Ronci, F. Silicon Reactivity at the Ag (111) Surface. Phys. Rev. Lett. 2015, 115, 026102 .

(28) Gao, J.; Zhao, J. Initial Geometries, Interaction Mechanism and High Stability of Silicene on Ag (111) Surface. Sci. Rep. 2012, 2, 861.

(29) Borensztein, Y.; Curcella, A.; Royer, S.; Prevot, G. Silicene Multilayers on Ag (111) Display a Cubic Diamond-Like Structure and a $\sqrt{3} \times \sqrt{3}$ Reconstruction Induced by Surfactant Ag Atoms. Phys. Rev. B: Condens. Matter Mater. Phys. 2015, 92, 155407.

(30) Guo, Z.-X.; Furuya, S.; Iwata, J.-I.; Oshiyama, A. Absence and Presence of Dirac Electrons in Silicene on Substrates. Phys. Rev. B: Condens. Matter Mater. Phys. 2013, 87, 235435.

(31) Kokott, S.; Pflugradt, P.; Matthes, L.; Bechstedt, F. Nonmetallic Substrates for Growth of Silicene: An Ab Initio Prediction. J. Phys.: Condens. Matter 2014, 26, 185002. 
(32) Kokott, S.; Matthes, L.; Bechstedt, F. Silicene on HydrogenPassivated Si (111) and Ge (111) Substrates. Phys. Status Solidi RRL 2013, 7, 538-541.

(33) Cai, Y.; Chuu, C.-P.; Wei, C. M.; Chou, M. Y. Stability and Electronic Properties of Two-Dimensional Silicene and Germanene on Graphene. Phys. Rev. B: Condens. Matter Mater. Phys. 2013, 88, 245408.

(34) De Crescenzi, M.; Piancastelli, M. N. Electron Scattering and Related Spectroscopies; World Scientific Publishing: Singapore, 1996; pp $1-415$.

(35) Santoni, A.; Frycek, R.; Castrucci, P.; Scarselli, M.; De Crescenzi, M. XPS and STM Study of SiC Synthesized by Acetylene and Disilane Reaction with the $\mathrm{Si}(100) 2 \times 1$ Surface. Surf. Sci. 2005, $582,125-136$.

(36) Friedlein, R.; Van Bui, H.; Wiggers, F. B.; Yamada-Takamura, Y.; Kovalgin, A. Y.; de Jong, M. P. Interaction of Epitaxial Silicene With Overlayers Formed by Exposure to $\mathrm{Al}$ Atoms and $\mathrm{O}_{2}$ Molecules. J. Chem. Phys. 2014, 140, 204705.

(37) Scheier, P.; Marsen, B.; Lonfat, M.; Schneider, W.-D.; Sattler, K. Growth of Silicon Nanostructres on Graphite. Surf. Sci. 2000, 458, 113-122.

(38) Sone, J.; Yamagami, T.; Nakatsuji, K.; Hirayama, H. Si Growth at Graphene Surfaces on 6H-SiC(0001) Substrates. Jpn. J. Appl. Phys. 2016, 55, 035502.

(39) Liu, C. C.; Feng, W.; Yao, Y. Quantum Spin Hall Effect in Silicene and Two-Dimensional Germanium. Phys. Rev. Lett. 2011, 107, 076802.

(40) Huang, B.; Deng, H.-X.; Lee, H.; Yoon, M.; Sumpter, B. G.; Liu, F.; Smith, S. C.; Wei, S.-H. Exceptional Optoelectronic Properties of Hydrogenated Bilayer Silicene. Phys. Rev. X 2014, 4, 021029.

(41) Kresse, G.; Furthmüller, J. Efficiency of $A b$ Initio Total Energy Calculations for Metals and Semiconductors Using a Plane-Wave Basis Set. Comput. Mater. Sci. 1996, 6, 15-50.

(42) Kresse, G.; Furthmüller, J. Efficient Iterative Schemes for $A b$ Initio Total-Energy Calculations Using a Plane-Wave Basis Set. Phys. Rev. B: Condens. Matter Mater. Phys. 1996, 54, 11169-11186.

(43) Perdew, J. P.; Burke, K.; Ernzerhof, M. Generalized Gradient Approximation Made Simple. Phys. Rev. Lett. 1996, 77, 3865-3868.

(44) Grimme, S. Semiempirical GGA-Type Density Functional Constructed with a Long-Range Dispersion Correction. J. Comput. Chem. 2006, 27, 1787-1799.

(45) Blöchl, P. E. Projector Augmented-Wave Method. Phys. Rev. B: Condens. Matter Mater. Phys. 1994, 50, 17953-17979.

(46) Windiks, R.; Delley, B. Massive Thermostatting in Isothermal Density Functional Molecular Dynamics Simulations. J. Chem. Phys. 2003, 119, 2481-2487.

(47) Nosé, S. A Unified Formulation of the Constant Temperature Molecular-Dynamics Methods. J. Chem. Phys. 1984, 81, 511-519. 